\title{
Proteomic Study of Mycoplasma Pneumoniae Pneumonia Reveals FCGBP as A Serum Biomarker and Implicates Potential Therapeutic Targets
}

\author{
Jinrong Liu ${ }^{1}$, Rongfang Shen ${ }^{2}$, Lin Feng ${ }^{2}$, Shujun Cheng ${ }^{2}$, Zhenrong Liu ${ }^{2}$, Jun Chen ${ }^{1}$, Ting \\ $\mathrm{Xiao}^{2}$, and shunying zhao ${ }^{3}$ \\ ${ }^{1}$ Beijing Children's Hospital Affiliated to Capital Medical University \\ ${ }^{2}$ National Cancer Center/National Clinical Research Center for Cancer/Cancer Hospital, \\ Chinese Academy of Medical Sciences and Peking Union Medical College \\ ${ }^{3}$ Bejing Childrens Hospital
}

June 9, 2020

\begin{abstract}
Macrolides and corticosteroid resistant have been reported in mycoplasma pneumoniae (MP) pneumonia (MPP). MP clearance is difficult even by sensitive antibiotics in severe MPP (SMPP). SMPP children might develop into airway remodeling even bronchiolitis/bronchitis obliterans. There is an urgent need to identify serum biomarkers indicating the progress of MPP and to discover new target drugs for the treatment of SMPP. In this study, we collected serum samples from general MPP (GMPP) and SMPP patients to perform proteomics profiling. Total 130 differentially expressed proteins with 61 up-regulated in GMPP and 69 up-regulated in SMPP were identified. Among these, FCGBP was one of the most altered protein with highest fold change. Biological enrichment analysis indicated an uncontrolled inflammation catastrophe in SMPP. In addition, complement, coagulation cascades, collagen-containing extracellular matrix and platelet degranulation pathway were enriched in both groups. KEGG analysis indicated an enriched platelet activation in SMPP. ELISA was then performed to verify the dynamic serum FCGBP expression level between other GMPP and SMPP patients. FCGBP level in SMPP was significantly higher than that in GMPP. FCGBP level in GMPP exhibited a decreased trend while SMPP showed the opposite trend during the disease course. Our study demonstrates the first proteomics characteristic of GMPP and SMPP and provides FCGBP as a new serum biomarker indicating the progress of SMPP. Further CMap analysis identified 25 drugs target for the treatment of SMPP. Among them, MTOR inhibitor, a macrolide compound and cell proliferation inhibitor, is the most promising drug targeting for the treatment of SMPP.
\end{abstract}

\section{Introduction}

The clinical presentation of mycoplasma pneumoniae (MP) pneumonia (MPP) includes pulmonary symptoms like bronchiolitis and pneumonia even necrotizing pneumonia, thrombosis, and the extra-pulmonary symptoms such as encephalitis, and hemolytic anemia. Persistent MP infection, airway obstruction and airway remodeling have been concerned in human airway epithelium, BALB/c mice, and SMPP children ${ }^{1-3}$. MPP patients may develop into severe life-threatening pneumonia (severe MPP, SMPP) with features of respiratory failure and acute respiratory distress syndrome, and sequelae such as atelectasis and bronchiectasis due to bronchiolitis/bronchitis obliterans (BO) resulting from airway remodeling ${ }^{2,4-7}$.

Due to the side effects of tetracyclines and quinolones, macrolides are used as first-line antibiotics for the treatment of MPP in young children. However, high presence of macrolide resistance has been reported and MP clearance is difficult even by sensitive antibiotics in refractory MPP (RMPP) and SMPP ${ }^{2}$. Evidence 
showed the incidence of SMPP has increased in recent years, particularly in East Asia, posing serious threat to children's health. Corticosteroid could effectively initiate the rapid improvement of clinical symptoms and chest radiographic findings, however its resistant has been reported in some RMPP patients with more severe presentations and more serious radiological finding ${ }^{8}$. In the near ten years, RMPP and SMPP are very common in North China particularly in our hospital, which have much higher sequelae rate mainly $\mathrm{BO}^{\mathbf{2 , 4 - 6}}$. Thus, there is an urgent need to discover serum biomarkers and target drugs for monitoring the progress and prevent airway remodeling of SMPP.

Numerous studies have been performed to explore biomarkers in order to detect RMPP or SMPP early. Several have been discovered for the severity evaluation of RMPP or SMPP in children, such as cytokines. However, variations and these biomarkers cannot predict severity or sequelae completely, limiting their clinical applications. In recent years, proteomics has emerged as a powerful method to investigate novel diagnostic and therapeutic targets. The consistency of peptide identification and protein sequence coverage in complex biological samples has been improved substantially with rapid advances in mass spectrometry technology. The label-free proteomic technology has been considered as a superior choice due to its high proteome coverage and labeling efficiency, particularly to obtain information not accessible using two-dimensional electrophoresis, and is employed widely in quantitative proteomics.

In the present study, initially we applied label-free proteomic analysis in general MPP (GMPP) and SMPP children, tried to find out differentially expressed proteins. Fc Fragment of IgG Binding Protein (FCGBP) was the identified protein with the highest expression difference and Enzyme-linked immunosorbent assay (ELISA) was then performed to verify the expression levels in GMPP and SMPP patients, respectively. In order to identify potential useful drugs that can be applied to the treatment of SMPP patients and deepen the understanding of the mechanism of SMPP, we adopt the Connectivity Map tool to identify potential useful drugs. To our knowledge, this is the first such study in the progress of MP infection related disease.

\section{Materials and methods}

\subsection{Clinical samples}

In total, forty-three MPP patients (17 GMPPs and 26 SMPPs) were enrolled in this study. In which, twenty patients (10 GMPPs and 10 SMPPs) received label free proteomic analysis and twenty- three patients (7 GMPPs and 16 SMPPs) underwent ELISA test. Serum samples, collected from patients between January 2016 and January 2017 in Beijing Children's Hospital, were stored and frozen at $-80^{\circ} \mathrm{C}$. Written informed consents were obtained by care givers of all children before entering the study. This study was approved by the Ethics Committee of Beijing Children's Hospital affiliated to Capital Medical University ((No. 2017-23).

\subsection{Diagnostic Criteria}

MPP was diagnosed based on the following criteria: (1) clinical presentation (fever, cough); (2) chest imaging with infiltrates; (3) serum anti-MP IgM titer [?]1:256 (double testing) or four-fold rising titer in acute and convalescent serum specimens. In addition, real-time PCR of MP deoxyribonucleic acid (DNA) in throat swabs was positive on the acute stage in all patients. In this study, SMPP was defined as MPP with the following symptoms: (1) pulse oxygen saturation in room air ?92\%, and (2) ?2/3 pulmonary lobe involvement. No other respiratory tract pathogens were detected in the enrolled patients. All patients received azithromycin therapy before sample collection and were previously healthy.

\subsection{Proteomic mass spectrometry}

Serum samples from GMPP and SMPP groups were pooled together separately and subjected to label-free proteomic technology to identify dysregulated proteins. Maxquant was implemented to process the raw mass spectrometry data. Proteins with false hits labeled by Maxquant including "potential contaminant", "reverse" and "only identified by site" were excluded. And only proteins detected in both groups were included in the subsequent analysis. Ultimately, we obtained 276 proteins satisfying above criteria. The LFQ intensity was used to represent the protein expression level. The raw data are publicly available on integrated Proteome resources center (iProX) under the accession number IPX0001738000. 


\subsection{Functional enrichment analysis}

The R package "clusterProfiler" ${ }^{9}$ was applied for the enrichment of Gene Ontology (GO) biological process and Kyoto Encyclopedia of Genes and Genomes (KEGG) pathway analysis. Enrichment significance was estimated using the default setting and 1000 permutations. Benjamini-Hochberg adjusted P-values less than 0.05 was considered as statistically significant. The KEGG pathway overview was implemented by the $\mathrm{R}$ package "pathview" 10.

\subsection{ELISA}

After identification of the targeted differentially expressed protein, FCGBP was found with the highest expression difference. ELISA was then implemented by following the manufacturer's kit instructions (AVIVA Systems Biology, San Diego, CA, USA) to verify circulating protein concentrations of FCGBP in GMPP and SMPP groups, respectively.

\subsection{Predication of transcription factors (TFs) regulating FCGBP}

We used Cistrome Data Browser toolkit (http://dbtoolkit.cistrome.org/) ${ }^{11}$ to predicate potential TFs that regulating targeted protein FCGBP. Cistrome Data Browser is a user-friendly web server for querying, exploring and visualizing ChIP-Seq and chromatin accessibility data. Since few ChIP-Seq data were publicly available in BEAS-2B cells, an epithelial cell line isolated from normal human bronchial epithelium, we query FCGBP's potential TFs in all available lung cells including A549, BEAS-2B and NCI-H2052 under the setting of $100 \mathrm{~kb}$ distance to transcription start site in order to include enhancer-type TFs.

\subsection{Connectivity Map Analysis}

Drug prediction, mechanism of actions (MoA) and drug-target analyses were performed on Connectivity Map (CMap) tools (https://clue.io/) ${ }^{12}$. CMap integrates gene expression signatures, drugs and disease states and is widely used to predict potential useful drugs, investigate the internal mechanism and interrelationship of drugs and inform clinical trials. The query and touchstone functions of CMap website were performed to drug prediction, MoA and drug-target analysis. Dysregulated genes between GMPP and SMPP including 47 up-regulated genes and 54 down-regulated genes were subjected to query tool. The "bitr" function of R package "cluster Profiler" was implemented to convert the uniport accessions into gene symbols. The R package "Complex Heatmap" 13 was used to visualize MoA and drug-target analyses results. Interaction relationship between the target genes of selected compounds was analyzed and visualized using STRING (https://string-db.org/) website.

\subsection{Statistical analysis}

All the statistical analyses were performed using $\mathrm{R}$ version 3.6.0 (https://www.r-project.org/). The T test was adopted to compare the significant difference level for continuous variables between GMPPs and SMPPs and Fisher's exact test was implemented in categorical variables. Proteins with absolute value of $\log 2$ fold change more than 0.5 between GMPP and SMPP were considered as differently expressed proteins. $\mathrm{P}$-value or Benjamini-Hochberg adjusted P-value of less than 0.05 when executing multiple comparisons was considered as statistical significance.

\section{Results}

\subsection{Characteristics of patients diagnosed with GMPP and SMPP}

A total 20 patients (from 4-years-5-months to 13-years-2-months) were enrolled in the label-free proteomic technology study. All the patients involved in the ELISA study were treated with azithromycin, corticosteroids, and bronchoscopy lavage therapy and followed up for at least 3 months. Table 1 shows the detailed clinical characteristics of patients enrolled in ELISA test (Table S1). Mean CRP level was $27 \mathrm{mg} / \mathrm{L}$, and 94 $\mathrm{mg} / \mathrm{L}$ in GMPP and SMPP patients at admission, respectively. Chest imaging showed consolidation with high density $>1 / 2$ pulmonary lobe in all GMPP patients and $>2 / 3$ lobe in all SMPP patients. At the early stage of disease, 2 patients (28.6\%) in GMPP group and 14 patients $(87.5 \%)$ in SMPP group showed mucus 
plug/thick mucus secretion in bronchoscopy, respectively; and 9 (56.2\%) SMPP patients appeared airway mucous necrosis. At the late stage, bronchoscopy revealed airway deformation due to airway remodeling in 12 SMPP patients (1 airway stenosis and 11 BO). No sequelae were observed in GMPP group. A significant statistical difference was observed in airway damage between the two groups $(\mathrm{P}<0.05)$.

\subsection{Identification of differentially expressed proteins and distinct biological processes between GMPP and SMPP}

Totally, we identified 130 differentially expressed proteins with 61 up-regulated in GMPP group and 69 up-regulated in SMPP group. Several inflammatory markers such as CRP, SAA1, and SAA2 were found to be up-regulated in SMPP group (Figure 1A). Biological processes such as humoral immune response, acute inflammatory response, protein activation cascade and receptor-mediated endocytosis were enriched in SMPP group (Figure 1B, Table S2). In contrast, enrichment of hydrolase activity, peptidase activity and plasma lipoprotein particles were observed in GMPP group (Figure 1C, Table S3). The KEGG analysis indicated an uncontrolled inflammation catastrophe in SMPP group with the evidence of pathways like complement and coagulation cascades, staphylococcus aureus infection, IL-17 and HIF-1 signaling pathway, and platelet activation were enriched (Figure 1D, Table S4-S5). The complement and coagulation cascades pathway were enriched both in GMPP and SMPP. Complement activation helps recruiting inflammatory and immunocompetent cells and cleaning the extraneous pathogens, and blood coagulation plays a crucial role in the regulation of blood pressure and sodium homeostasis. Figure 1E shows the overview of complement and coagulation cascades pathway alternation between GMPP and SMPP. Many components involving coagulation system including coagulation factors such as F2, F5, F9, F13 and fibrinogen were up-regulated in SMPP group, while complement components C2, C4 and cluster in were altered in GMPP group.

\subsection{FCGBP is a novel indicator for predicting the progress of SMPP}

Among all the identified proteins which were differentially expressed, FCGBP was one of the most altered protein with highest fold change. We then used ELISA to explore its serum expression level between GMPP and SMPP patients, and tried to investigate the relationship between FCGBP level and the progress of MPP. On the first day of admission, SMPP patients showed a higher FCGBP expression tendency $(88.22+-$ $17.48 \mathrm{ng} / \mathrm{mL}, \mathrm{n}=16)$ than that in GMPP patients $(50.97+-10.24 \mathrm{ng} / \mathrm{mL}, \mathrm{n}=7)$ but did not reach a statistic significance (Figure $2 \mathrm{~A}, \mathrm{P}=0.057$ ). In the late stage of disease, SMPP patients exhibited a significantly higher FCGBP expression than the GMPP patients (Figure 2B, $\mathrm{P}=0.0039$ ). During the disease course, the expression level of serum FCGBP in GMPP group exhibited a decreased trend while the SMPP group showed the opposite trend after appropriate treatment (Figure 2C, 2D). Severe sequelae like BO tend to occur at SMPP patients. These data implicated that FCGBP may be a promising indicator for predicting the progression of SMPP and patients who maintain high serum level of FCGBP during treatment may have a poor prognosis comparing with who decrease.

\subsection{Potential TFs regulating FCGBP expression}

The mechanism under the dysregulated expression of FCGBP remains poorly illuminated. Since TF controls the correct gene expression from DNA to message RNA, we subjected FCGBP to Cistrome Data Browser toolkit in order to predict potential TFs, detailed information was described in method section. Figure 3 shows the identified TFs.

\subsection{Identification of potential SMPP therapeutic drugs including MTOR inhibitor}

We implemented CMap, the world's largest perturbation-driven gene expression dataset, to find perturbagens giving rise to opposing expression signature as candidate therapeutic drugs. Connectivity score is a holistic quantification index evaluating the similarity between the query signature and reference metagenes. Top 10 compounds with most positive and negative connectivity score are showed in Figure $4 \mathrm{~A}$. We chose compounds whose connectivity score was less than -70 as candidate drugs (full results showed in Table S6). In total, 25 drugs were selected for further analysis. CMap mode-of-action (MoA) analysis of these 25 drugs showed 27 potential underlying mechanism (Figure 4B). Except for commonly known drug mechanisms like 
anti-inflammatory and acetylcholine receptor antagonist, MTOR inhibitor was also identified. Gene target analysis of these 25 drugs revealed 54 target genes (Figure 4C). Although no overlap between these target genes was found, protein-protein interaction analysis indicated that they were highly connected to each other (Figure 4D).

\section{Discussion}

In recent years, with the development of techniques, increasing high throughput sequencing was used in MP studies. Lluch-Senar et al performed a comparative "-omics" analysis and revealed more toxigenic of type 2 strains than type 1 strains of MP due to higher expression levels of CARDS toxin ${ }^{14}$. By using RNA sequencing in bronchoalveolar lavage fluid, Gao M et al found 810 differentially expressed genes in MPP group comparing to control group, suggesting NK and CD8+ T cells are over activated and proliferated in MPP children ${ }^{15}$. Proteomics is now increasingly used to explore biomarkers of infectious diseases, which can provide us a feasible mean for large-scale screening of SMPP-related proteins, thus enhancing our understanding of the pathogenesis of SMPP. However, till now only two studies performed proteomics analysis in $\mathrm{MPP}^{16,17}$, one used serum samples from 5 RMPP children, 5 non-RMPP children, and 5 healthy children, while the other selected plasma from children with MPP, infectious disease controls and healthy controls. There are very few reports on proteomics analysis of SMPP. To our knowledge, ours is the first proteomics study in GMPPs and SMPPs to explore marker for dynamic progress of SMPP.

In the present study, by using label-free quantitative analysis, we initially explored differentially expressed proteins between GMPP and SMPP children and found 130 proteins. Interestingly, the complement and coagulation cascades pathway were enriched in both GMPP and SMPP groups, which might be explained by the varied pathway activation extent. Since the moderate activation of complement and coagulation cascades pathway assisted in cleaning microbial intruders, it is convincing that organism strived to clean pathogens and retain homeostasis in GMPP through activating the pathway. While dysregulation of the acute phase involving cytokine storm can result in severe clinical symptoms like acute lung injury ${ }^{18}$, hypercoagulable stat even thrombosis ${ }^{4}$, thus the disorder of complement and coagulation cascades pathway might play a vital role in the incidence of SMPP. In addition, collagen-containing extracellular matrix and platelet degranulation were enriched in both GMPP and SMPP groups (Figure 1B 、1C), and KEGG analysis indicated an enriched platelet activation in SMPP group (Figure 1D), which might be explained by the varied airway remodeling and platelet activation extent. Typical characteristics of SMPP includes excessive immune response, hypercoagulable stat even thrombosis, and subsequent airway remodeling such as airway stenosis and $\mathrm{BO}^{4}$. It is worth noting that platelets are key mediators of inflammatory responses. Platelets and the coagulation pathway activate innate immune cells ${ }^{19}$. Active platelets may regulate pulmonary immune defenses and inflammatory injury ${ }^{20}$, immunothrombosis ${ }^{21}$, and is necessary in airway wall remodeling ${ }^{22}$. Therefore, the disorder of platelet activation might play another vital role in SMPP.

Among all the identified differentially expressed proteins including CRP and SAA, FCGBP was one of the most altered protein with highest fold change. FCGBP may play a role in the maintenance of the mucosal structure as a gel-like component of the mucosa. It can covalently bind and cross-link mucus proteins via its autocatalytically cleaved von Willebrand D domain ${ }^{23}$. Differentially expression of FCGBP has been reported gallbladder, prostate, thyroid, lung ${ }^{24}$, colon, and ovarian cancer. Present in serum, the protein can be found in higher levels in patients with autoimmune diseases ${ }^{25}$. Of special note, FCGBP has been postulated to trap HIV-1-antibody complexes at mucosal surfaces ${ }^{26}$. The expression of FCGBP was assumed to be modulated by HPV infection ${ }^{27}$ and it was the highest upregulated early defense gene in catfish skin after microbial infection ${ }^{28}$. In recent years, FCGBP has been reported to bind trefoil factor family 3 (TFF3), forming heterodimeric complex ${ }^{29}$. Interestingly, TFF3 has been reported a lectin activity, enabling binding to bacterial glycans, such as lipopolysaccharide of $\mathrm{H}$. pylori and exhibiting potential antibacterial activities ${ }^{30,31}$. Therefore, it has been considered that FCGBP was a component of first line responder in innate immunological defense, critically regulating pathogen attachment and disease progression in mucosal surface, thus playing roles in cell protections and anti-inflammation in tissues.

In our study, in the early stage, patients with SMPP showed a higher FCGBP expression tendency than 
that in GMPP group, but did not reach a statistic significance, which may be due to the acute stage of the disease or the relatively severe in GMPP patients who had $>1 / 2$ pulmonary lobe consolidation on chest imaging in our hospital. On the late stage, GMPP patients got reviled and did not need more FCGBP, thus the expression level reduced. However, for SMPP patients, even CRP level restored to baseline, the body temperature returned to normal, the airway damage did not relieved, even airway deformation such as $\mathrm{BO}$ occurred, which suggested airway epithelial cell and fibroblastic proliferation, and airway remodeling, and more FCGBP was produced to play anti-inflammation, anti-proliferation, anti-remodeling and cell protection roles. To some extent, these results indicated that FCGBP could be a biomarker for progression of MPP or airway remodeling in MPP. Since we only included 23 patients in our ELISA analysis, well designed and large cohort of randomized controlled trial will be needed before clinical application.

FCGBP is a key regulator in the process of transforming growth factor $\beta 1$ (TGF- $\beta 1$ ) induced epithelialmesenchymal transition (EMT) in gallbladder cancer ${ }^{32}$. TGF- $\beta$ is a platelet-granule constituent ${ }^{21}$ and airway $^{2}$ remodeling is consistent with EMT in MP infection ${ }^{1}$. Thus, we think FCGBP and platelet activation are very important in airway remodeling of SMPP. Among the identified TFs, TCF7L1 ranks top 3, which is a member of the $\mathrm{T}$ cell factor/lymphoid enhancer factor family of TFs and involves in the wnt signaling pathway to allow human stem cell differentiation precisely ${ }^{33,34}$. And a study based on smallpox vaccine response genome-wide association indicated that TCF7L1 is critical for lymphocyte IFN $\gamma$ production or cytotoxic $\mathrm{T}$ cell function ${ }^{35}$, showing its important role in regulating inflammatory response and eradicating foreign pathogens. Besides, numerous studies have linked the activation of CEBPB and RELA with immune and inflammatory responses, implicating they might orchestrate with FCGBP for the development of SMPP. RELA is a subunit of NF-kB, which is a major transcriptional regulator of genes involved in survival, proliferation and inflammation. NF-kB activation has been reported in MP infection ${ }^{36}$. Our results suggest that these TFs might regulate the expression of FCGBP, thus promote the development and increase the severity of SMPP.

Anti-inflammatory treatment mainly glucocorticoid is extremely important in SMPP. However, glucocorticoid resistance has been found in many SMPP patients ${ }^{8}$. Comparing to SMPP, steroid binding was enriched in GMPP (Figure 1C), which might predict a good prognosis. In addition, large amounts of SMPP patients miss their best glucocorticoid therapeutic opportunity in the early stage. Besides, glucocorticoid had side effects like osteoporosis and increased risk of secondary infection. As patients developing into SMPP would lead to respiratory failure, massive pleural effusion, atelectasis, necrotizing pneumonia, even subsequent BO, and even life-threatening, finding drugs preventing GMPP from developing into SMPP is of critical importance. In our study, 25 drugs were predicted, which may give us more chance to treat the disease early and effectively. MTOR inhibitor, sirolimus (rapamycin), which was also identified. Vascular endothelial growth factor (VEGF) released by endothelial cells induces angiogenesis and its elevated expression level is observed in some MPP $\mathrm{MP}^{37}$ and SMPP patients (unpublished data). MTOR inhibitor, an immunosuppressive agent and a macrolide compound, was isolated in 1975 fromStreptomyces hygroscopicus in a soil sample from Easter Island, which may effectively suppress inflammation and angiogenesis including VEGF, and block T-cell activation and proliferation. Sirolimus may be useful for the treatment of acute lung injury ${ }^{38}$ and decreases airway remodeling caused by TGF $-\alpha$ induced/EGF receptor-mediated signaling ${ }^{38}$. The TOR pathway regulates morphogenesis and responses to host cells in the fungal pathogen Candida albicans ${ }^{39}$, which suggests it may also regulate the responses to host cells in MPP. Low dosages of sirolimus had a good profile of safety and tolerability in pediatric patients ${ }^{40}$. Considering the evidence of angiogenesis, excessive inflammatory response, T cell-proliferation and airway remodeling in SMPP, we highly suspect that sirolimus, a macrolide antibiotic, is a promising drug target for the treatment of SMPP. Further investigations about the drug sensitivity test to MP, the mechanisms of MTOR inhibitor in the treatment of SMPP and randomized clinical trial are needed to promote its clinical usage.

\section{Conclusions}

Our study demonstrates the first proteomics characteristic of SMPP and GMPP and provides FCGBP as new serum biomarker indicating the progress of SMPP. In addition, sirolimus, a MTOR inhibitor, is a promising 
drug target for the treatment of SMPP.

Acknowledgments. We would like to thank all patients who donate their serum sample selflessly.

Disclaimer. The funders had no roles in study design, data collection and analysis, decision to publish, or preparation of the manuscript.

Funding. This study was supported by CAMS Innovation Fund for Medical Sciences (CIFMS) (Grant No. 2019-I2M-1-003), Natural Science Foundation of China (81741060) and Beijing Municipal Natural Science Foundation (7182051).

Conflict of interest statement. All authors declare that there is no conflict of interest.

Figure legends

Figure 1. Biological changes between GMPP and SMPP

(A) 2D scatter plot of the identified proteins which are detached in both GMPP and SMPP group. Proteins with $\log 2 \mathrm{FC}$ more than 0.5 are thought as different expressed proteins and are colored with blue or red. Corresponding gene symbols of those proteins with $\log 2 \mathrm{FC}$ more than 1.5 are labeled in the plot. (B, C) GO enrichment analysis results of the dysregulated genes which are enriched in SMPP group (B) and GMPP group (C). Top 15 with most significant enrichment results of each category (biological process, molecular function and cellular component) are showed. (D) KEGG enrichment analysis results of the dysregulated genes which are enriched in SMPP group (top) and GMPP group (bottom). All enrichment terms which reach enrichment significance are showed. (E) The overview of complement and coagulation cascades pathway. Genes in the box with color (green, grey and red) are the identified proteins in this pathway. The color intensity represents the corresponding log2FC level, increasing color intensity indicates that increasing enrichment level in SMPP group (red) and GMPP group (green).

Figure 2. FCGBP is a promising indicator for the progress of SMPP

The serum concentration of FCGBP identified by ELISA between GMPP and SMPP group at the first day of admission (A) and the 5-7 day of admission (B). The statistical significance level of pairwise comparisons is annotated with text. (C). Pairwise FCGBP serum concentration of GMPP group between the treatment naïve patients at the first day of admission and the 5-7 day of admission. (D). Pairwise FCGBP serum concentration of SMPP group between the treatment naïve patients at the first day of admission and the 5-7 day of admission. The statistical significance level of pairwise comparisons is annotated with text.

Figure 3 Potential TFs that regulates FCGBP expression.

Top $20 \mathrm{TFs}$ with the highest regulatory potential score are showed. Regulatory potential score estimates the possibility that the TF can regulate a gene.

Figure 4. Promising therapeutic drugs that inhibit the progress of SMPP

(A) Bar plot of candidate perturbations inferred from connectivity map analysis. Top 10 compounds with most positive and negative connectivity score are showed. (B) CMap mode-of-action (MoA) analysis under the selected 25 drugs whose connectivity scores less than -70. Potential mechanisms and inhibitors are placed on the right and the top panel. The perturbations' mode of actions are indicated with black dots. (C) CMap gene target analysis under the selected 25 drugs. Identified target genes and inhibitors are placed on the right and top panel. The perturbations' target genes are indicated with black dots. (D) Protein-protein interaction network of the target genes inferred from CMap gene target analysis. The relationships between these proteins are inferred from STRING.

\section{REFERENCES}

1. Prince OA, Krunkosky TM, Sheppard ES, Krause DC. Modelling persistent Mycoplasma pneumoniae infection of human airway epithelium. Cell Microbiol 2018;20. 
2. Liu J, Zhao F, Lu J, Xu H, Liu H, Tang X, Yang H, Zhang J, Zhao S. High Mycoplasma pneumoniae loads and persistent long-term Mycoplasma pneumoniae DNA in lower airway associated with severity of pediatric Mycoplasma pneumoniae pneumonia. BMC Infect Dis 2019;19:1045.

3. Salvatore CM, Fonseca-Aten M, Katz-Gaynor K, Gomez AM, Hardy RD. Intranasal interleukin-12 therapy inhibits Mycoplasma pneumoniae clearance and sustains airway obstruction in murine pneumonia. Infect Immun 2008;76:732-738.

4. Liu J, He R, Wu R, Wang B, Xu H, Zhang Y, Li H, Zhao S. Mycoplasma pneumoniae pneumonia associated thrombosis at Beijing Children's hospital. BMC Infect Dis 2020;20:51.

5. Zhao C, Liu J, Yang H, Xiang L, Zhao S. Mycoplasma pneumoniae-Associated Bronchiolitis Obliterans Following Acute Bronchiolitis. Sci Rep 2017;7:8478.

6. Liu JR, Lu J, Dong F, Li HM, Liu H, Tang XL, Guo YL, Zhao SY. Low Bacterial Co-infection Invalidates the Early Use of Non-anti-Mycoplasma pneumoniae Antibiotics in Pediatric Refractory Mycoplasma pneumoniae Pneumonia Patients. Front Pediatr 2018;6:296.

7. Leong MA, Nachajon R, Ruchelli E, Allen JL. Bronchitis obliterans due to Mycoplasma pneumonia. Pediatr Pulmonol 1997;23:375-381.

8. Yan Y, Wei Y, Jiang W, Hao C. The clinical characteristics of corticosteroid-resistant refractory Mycoplasma Pneumoniae pneumonia in children. Sci Rep 2016;6:39929.

9. Yu G, Wang LG, Han Y, He QY. clusterProfiler: an R package for comparing biological themes among gene clusters. OMICS 2012;16:284-287.

10. Luo W, Brouwer C. Pathview: an R/Bioconductor package for pathway-based data integration and visualization. Bioinformatics 2013;29:1830-1831.

11. Zheng R, Wan C, Mei S, Qin Q, Wu Q, Sun H, Chen CH, Brown M, Zhang X, Meyer CA, et al. Cistrome Data Browser: expanded datasets and new tools for gene regulatory analysis. Nucleic Acids Res 2019;47:D729-729D735.

12. Subramanian A, Narayan R, Corsello SM, Peck DD, Natoli TE, Lu X, Gould J, Davis JF, Tubelli AA, Asiedu JK, et al. A Next Generation Connectivity Map: L1000 Platform and the First 1,000,000 Profiles. Cell 2017;171:1437-1452.e17.

13. Gu Z, Eils R, Schlesner M. Complex heatmaps reveal patterns and correlations in multidimensional genomic data. Bioinformatics 2016;32:2847-2849.

14. Lluch-Senar M, Cozzuto L, Cano J, Delgado J, Llórens-Rico V, Pereyre S, Bebear C, Serrano L. Comparative "-omics" in Mycoplasma pneumoniae Clinical Isolates Reveals Key Virulence Factors. PLoS One 2015;10:e0137354.

15. Gao M, Wang K, Yang M, Meng F, Lu R, Zhuang H, Cheng G, Wang X. Transcriptome Analysis of Bronchoalveolar Lavage Fluid From Children With Mycoplasma pneumoniae Pneumonia Reveals Natural Killer and T Cell-Proliferation Responses. Front Immunol 2018;9:1403.

16. Li J, Sun L, Xu F, Qi H, Shen C, Jiao W, Xiao J, Li Q, Xu B, Shen A. Screening and Identification of APOC1 as a Novel Potential Biomarker for Differentiate of Mycoplasma pneumoniae in Children. Front Microbiol 2016;7:1961.

17. Yu JL, Song QF, Xie ZW, Jiang WH, Chen JH, Fan HF, Xie YP, Lu G. iTRAQ-based Quantitative Proteomics Study in Patients with Refractory Mycoplasma pneumoniae Pneumonia. Jpn J Infect Dis 2017;70:571-578.

18. Abraham E. Coagulation abnormalities in acute lung injury and sepsis. Am J Respir Cell Mol Biol 2000;22:401-404. 
19. Engelmann B, Massberg S. Thrombosis as an intravascular effector of innate immunity. Nat Rev Immunol 2013;13:34-45.

20. Fox KA, Kirwan DE, Whittington AM, Krishnan N, Robertson BD, Gilman RH, López JW, Singh S, Porter JC, Friedland JS. Platelets Regulate Pulmonary Inflammation and Tissue Destruction in Tuberculosis. Am J Respir Crit Care Med 2018;198:245-255.

21. Middleton EA, Weyrich AS, Zimmerman GA. Platelets in Pulmonary Immune Responses and Inflammatory Lung Diseases. Physiol Rev 2016;96:1211-1259.

22. Pitchford SC, Riffo-Vasquez Y, Sousa A, Momi S, Gresele P, Spina D, Page CP. Platelets are necessary for airway wall remodeling in a murine model of chronic allergic inflammation. Blood 2004;103:639-647.

23. Johansson ME, Thomsson KA, Hansson GC. Proteomic analyses of the two mucus layers of the colon barrier reveal that their main component, the Muc2 mucin, is strongly bound to the Fcgbp protein. J Proteome Res 2009;8:3549-3557.

24. Zhou C, Chen H, Han L, Xue F, Wang A, Liang YJ. Screening of genes related to lung cancer caused by smoking with RNA-Seq. Eur Rev Med Pharmacol Sci 2014;18:117-125.

25. Kobayashi K, Yagasaki M, Harada N, Chichibu K, Hibi T, Yoshida T, Brown WR, Morikawa M. Detection of Fcgamma binding protein antigen in human sera and its relation with autoimmune diseases. Immunol Lett 2001;79:229-235.

26. Schwartz JL. Fcgbp - A Potential Viral Trap in RV144. Open AIDS J 2014;8:21-24.

27. Wang Y, Liu Y, Liu H, Zhang Q, Song H, Tang J, Fu J, Wang X. FcGBP was upregulated by HPV infection and correlated to longer survival time of HNSCC patients. Oncotarget 2017;8:86503-86514.

28. Li C, Wang R, Su B, Luo Y, Terhune J, Beck B, Peatman E. Evasion of mucosal defenses during Aeromonas hydrophila infection of channel catfish (Ictalurus punctatus) skin. Dev Comp Immunol 2013;39:447-455.

29. Houben T, Harder S, Schlüter H, Kalbacher H, Hoffmann W. Different Forms of TFF3 in the Human Saliva: Heterodimerization with IgG Fc Binding Protein (FCGBP). Int J Mol Sci 2019;20.

30. Reeves EP, Ali T, Leonard P, Hearty S, O'Kennedy R, May FE, Westley BR, Josenhans C, Rust M, Suerbaum S, et al. Helicobacter pylori lipopolysaccharide interacts with TFF1 in a pH-dependent manner. Gastroenterology 2008;135:2043-2054, 2054.e1-2.

31. Dolan B, Naughton J, Tegtmeyer N, May FE, Clyne M. The interaction of Helicobacter pylori with the adherent mucus gel layer secreted by polarized HT29-MTX-E12 cells. PLoS One 2012;7:e47300.

32. Xiong L, Wen Y, Miao X, Yang Z. NT5E and FcGBP as key regulators of TGF-1-induced epithelialmesenchymal transition (EMT) are associated with tumor progression and survival of patients with gallbladder cancer. Cell Tissue Res 2014;355:365-374.

33. Morrison G, Scognamiglio R, Trumpp A, Smith A. Convergence of cMyc and $\beta$-catenin on Tcf7l1 enables endoderm specification. EMBO J 2016;35:356-368.

34. Chen Z, Ji Z, Ngiow SF, Manne S, Cai Z, Huang AC, Johnson J, Staupe RP, Bengsch B, Xu C, et al. TCF-1-Centered Transcriptional Network Drives an Effector versus Exhausted CD8 T Cell-Fate Decision. Immunity 2019;51:840-855.e5.

35. Kennedy RB, Ovsyannikova IG, Pankratz VS, Haralambieva IH, Vierkant RA, Jacobson RM, Poland GA. Genome-wide genetic associations with IFN $\gamma$ response to smallpox vaccine. Hum Genet 2012;131:1433-1451.

36. Zhao Y, Ma G, Yang X. HDAC5 promotes Mycoplasma pneumoniae-induced inflammation in macrophages through NF- $x$ B activation. Life Sci 2019;221:13-19. 
37. Choi IS, Byeon JH, Yoo Y, Lee KC, Choung JT. Increased serum interleukin-5 and vascular endothelial growth factor in children with acute mycoplasma pneumonia and wheeze. Pediatr Pulmonol 2009;44:423-428.

38. Wang SH, Li LH, Zou DM, Zheng XM, Deng J. Roles of the mammalian target of rapamycin (mTOR) signaling pathway in the repair of hyperoxia-induced acute lung injury. Adv Clin Exp Med 2020;29:13-23.

39. Liu NN, Flanagan PR, Zeng J, Jani NM, Cardenas ME, Moran GP, Köhler JR. Phosphate is the third nutrient monitored by TOR in Candida albicans and provides a target for fungal-specific indirect TOR inhibition. Proc Natl Acad Sci U S A 2017;114:6346-6351.

40. Bevacqua M, Baldo F, Pastore S, Valencic E, Tommasini A, Maestro A, Rabusin M, Arbo A, Barbi E. Off-Label Use of Sirolimus and Everolimus in a Pediatric Center: A Case Series and Review of the Literature. Paediatr Drugs 2019;21:185-193.

\section{Hosted file}

Table 1.docx available at https://authorea.com/users/322398/articles/458205-proteomic-studyof-mycoplasma-pneumoniae-pneumonia-reveals-fcgbp-as-a-serum-biomarker-and-implicatespotential-therapeutic-targets 
A

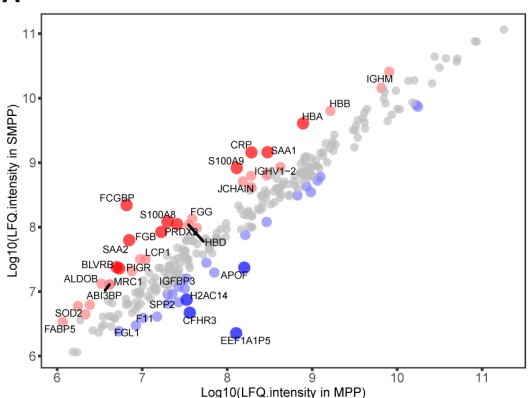

C

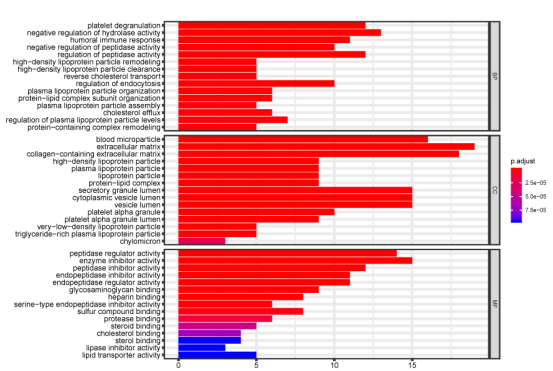

B

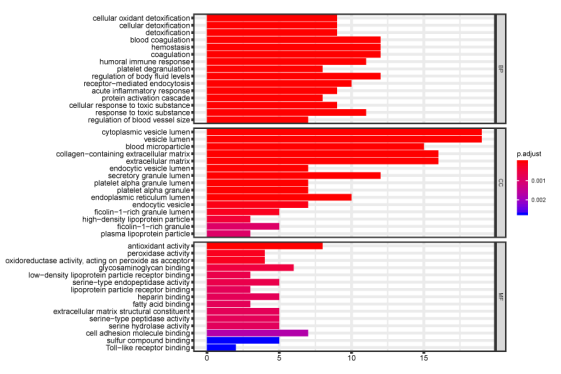

D

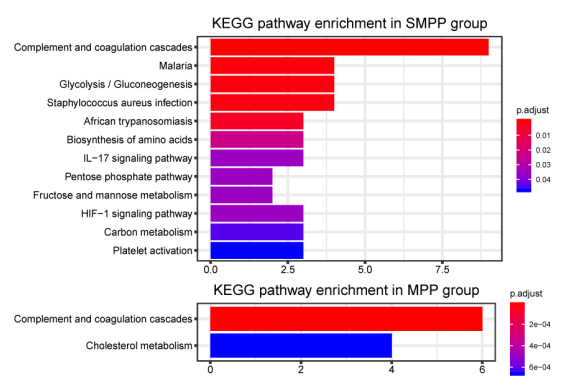

E

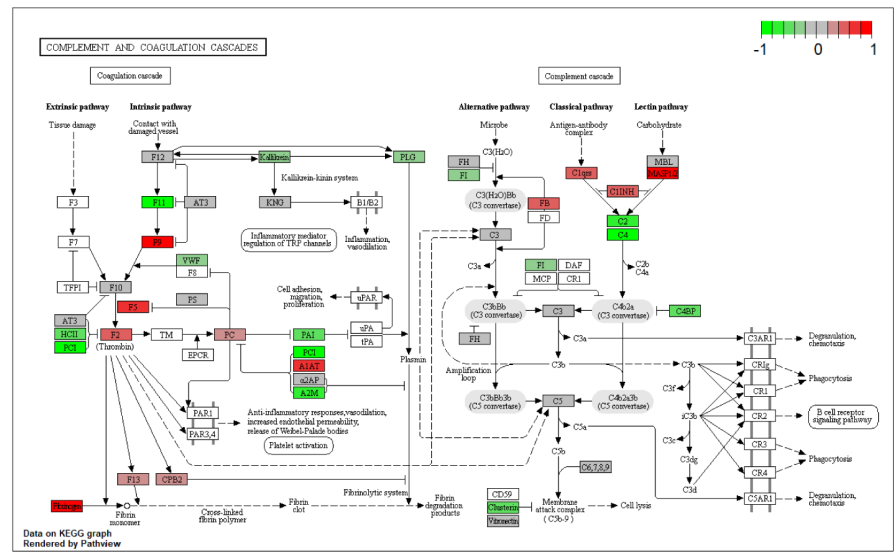


A

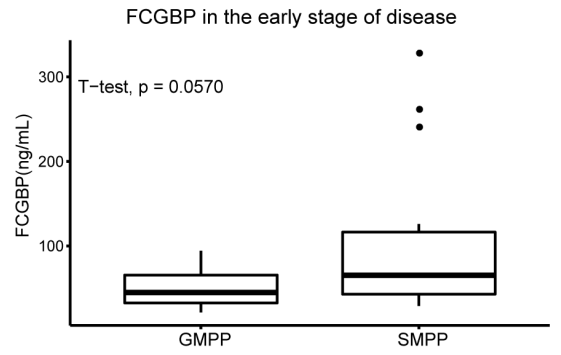

C

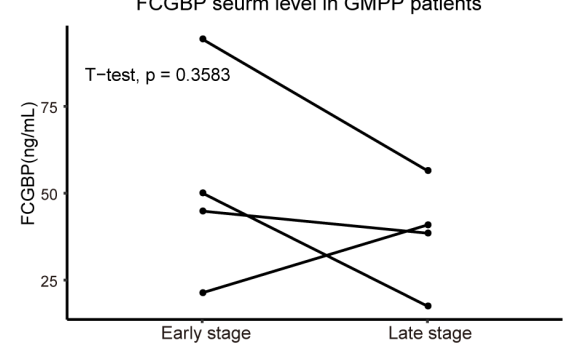

B

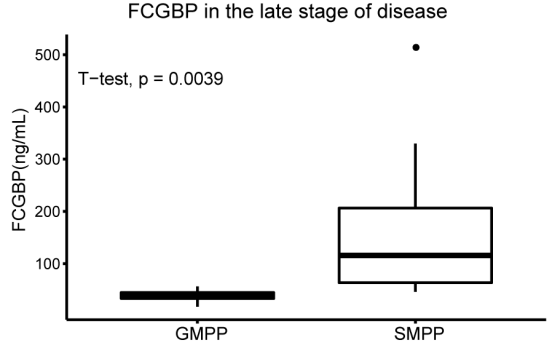

D

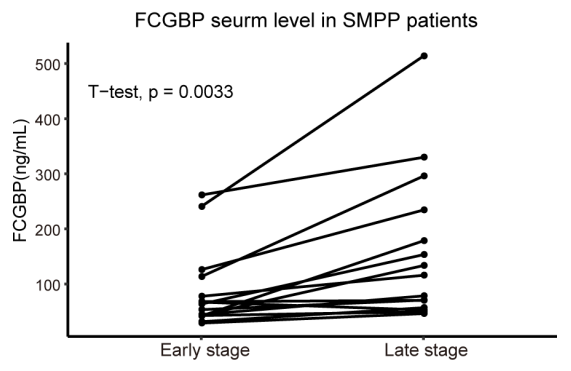

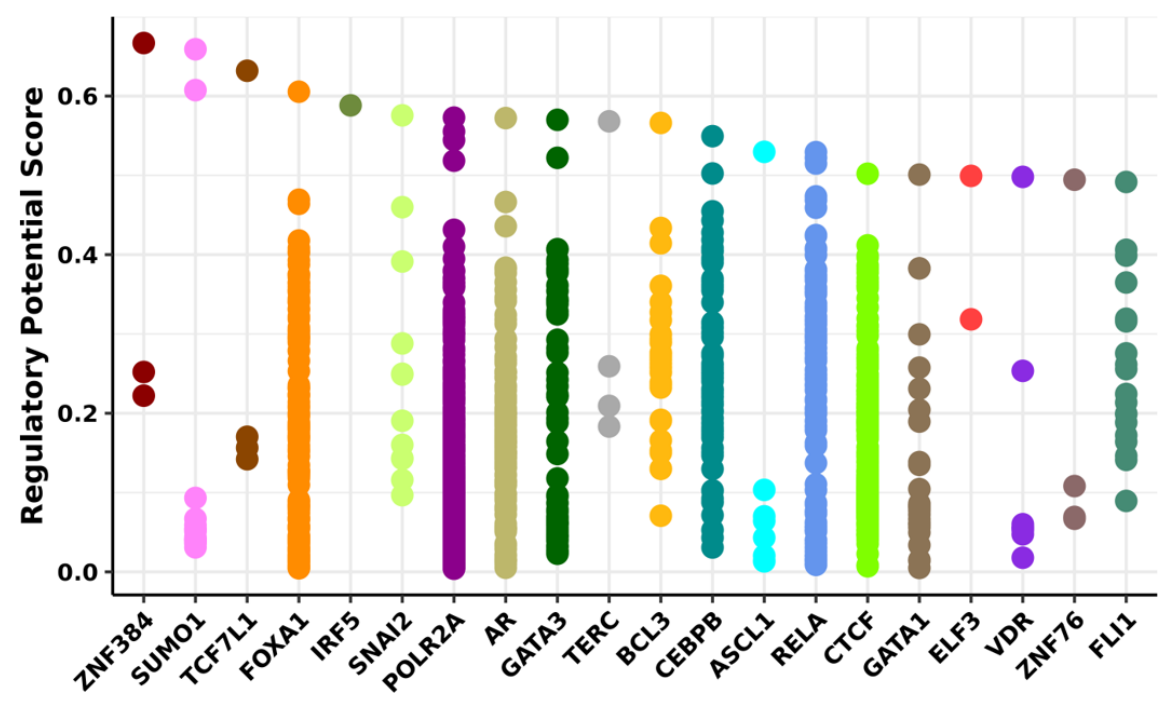



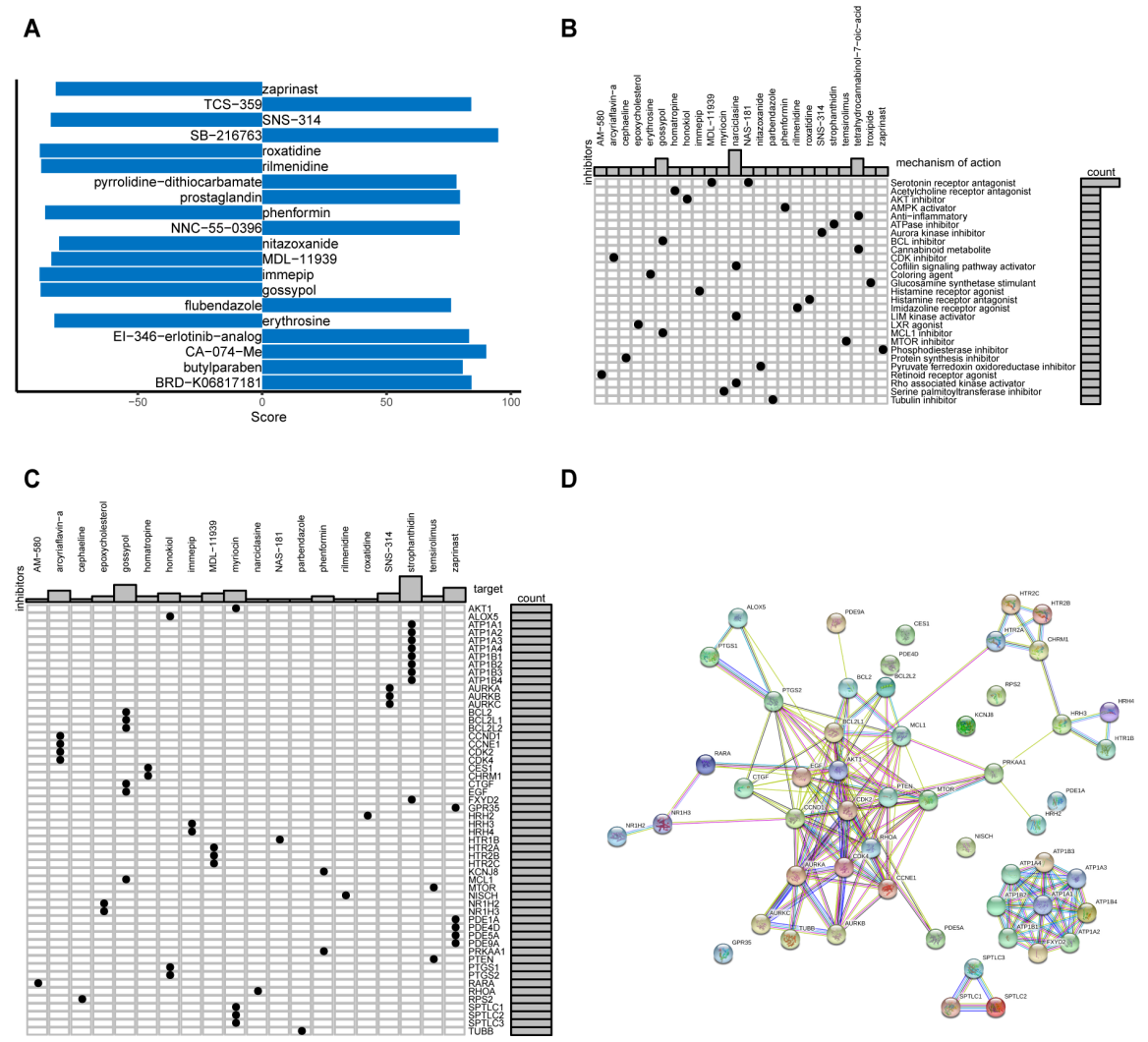